\title{
The synergistic effect of homocysteine and lipopolysaccharide on the differentiation and conversion of raw264.7 macrophages
}

\author{
Shanshan Gao, Lijun Wang, Weimin Liu, Yue Wu and Zuyi Yuan*
}

\begin{abstract}
Background: Macrophages play pivotal roles in the progression of atherosclerosis (AS) and their heterogeneous differentiation patterns have been studied extensively. The classical subtype of activated macrophage, M1, promotes the progression of AS. Conversely, the alternative subtype of activated macrophage, M2, is regarded as a repressor of AS. Homocysteine (Hcy) may influence macrophage subtype polarization both in vivo and in vitro. Homocysteinemia (HHcy) is an independent risk factor in coronary heart disease and the effect of Hcy on macrophage differentiation has not been studied until now.

Methods: Different concentrations of Hcy in combination with a fixed concentration of lipopolysaccharide (LPS, $200 \mathrm{ng} / \mathrm{mL}$ ) were used to treat RAW264.7 macrophages. Real-time PCR was used to detect and quantify RNA transcripts indicative of $\mathrm{M} 1$ and $\mathrm{M} 2$ differentiation. The efficacy and specificity for each chemical stimulant in inducing macrophage differentiation were also investigated. The M2 macrophages (anti-inflammatory subtype) induced using classical methods (IL-4, $10 \mathrm{ng} / \mathrm{mL}$ ) were also treated with different concentrations of Hcy complemented with LPS. The synergistic effect of Hcy and LPS in the converting the M2 subtype to M1 was also studied.

Results: Macrophages can be induced to differentiate towards M1 by a combination of Hcy with LPS, with the strongest effect observed at an Hcy concentration of $50 \mu \mathrm{mol} / \mathrm{L}$. After inducing macrophages to the M2 subtype using IL-4, treatment with both Hcy and LPS could elicit conversion from the M2 to M1 subtype.

Conclusion: Combined treatment with Hcy and LPS can induce the polarization of cultured RAW264.7 macrophages into the pro-inflammatory subtype, as well as promote subtype conversion from anti-inflammatory to pro-inflammatory.
\end{abstract}

Keywords: Homocysteine, Lipopolysaccharide, Macrophages, M1/M2 subtype, Polarization, Subtype conversion

\section{Background}

Atherosclerosis (AS) is a chronic and progressive inflammatory disease characterized by the accumulation of lipids and fibrous lesions in the large arterial intima. Mechanisms governing AS continue to draw extensive research interest. Among proposed mechanisms, the immuno-inflammation hypothesis has become accepted by most researchers [1-3]. Macrophages are important in both the initiation and progression of AS $[4,5]$. Macrophages can differentiate into two subtypes (M1 and M2) in response to microenvironmental changes. Considered the classical activated

\footnotetext{
* Correspondence: zuyiyuan@mail.xjtu.edu.cn

Department of Cardiology, the First Affiliated Hospital of Medical College, Xi'an Jiaotong University, Xi'an 710061, China
}

subtype, M1 exerts pro-inflammatory effects that promote the progression of AS. In contrast, the M2 activated subtype exerts anti-inflammatory effects and is regarded as a repressor of AS [6,7].

Hyperhomocysteinemia (HHcy, abnormally high physiological levels of homocysteine, Hcy) is regarded as an independent risk factor in coronary heart disease, although the exact mechanism of how Hcy induces AS is unclear. Studies have shown that Hcy can promote AS occurrence and progression through oxidative stress damage, endothelial damage, and activation of inflammatory and immune responses [8,9]. A positive correlation between HHcy, endothelial dysfunction, and accelerated AS has been demonstrated by using gene-induced and diet-induced HHcy 
animal models [10]. The results of some studies also reveal that Hcy could induce monocyte/macrophage localization in inflammatory lesions and exert pro-AS effects. Additionally, researchers have found that Hcy can influence the differentiation of macrophages both in vivo and in vitro [11]. However, it is unclear if the effect of Hcy on macrophage polarization and subtype conversion correlates to its pathogenic role in AS.

\section{Materials and methods \\ Cell culture}

The RAW264.7 macrophage cell line was provided by the Cardiology Institute of the First Affiliated Hospital of Medical College, Xi'an Jiaotong University [12]. RAW264.7 cells were cultured in DMEM supplemented with $10 \%$ (vol/vol) FBS, penicillin $(100 \mathrm{U} / \mathrm{mL})$ and streptomycin $(100 \mu \mathrm{g} / \mathrm{mL})$. In a 6 -well round bottom plate, $1 \times 10^{6}$ cells per well were seeded and incubated at $37^{\circ} \mathrm{C}$ for 12 hours. Before stimulation by Hcy and LPS, cells were serum-starved by culturing in DMEM without 10\% FBS for 12 hours.

\section{Reagents}

Hcy and LPS were from Sigma (Milwaukee, USA). IL-4 was from Peprotech (Rocky Hill, USA). The upstream and downstream primers of GAPDH, IL-6, IL-10, TNF- $\alpha$, iNOS, Arg-1, and MMR (shown in Table 1) were synthesized by Beijing Augct Biotechnology Co., Ltd. (Beijing, China). The RNA Fast 200 kit was from Shanghai Flytech Biotechnology Co., Ltd (Shanghai, China). The RevertAid ${ }^{\mathrm{TM}}$ First Strand cDNA Synthesis Kit was from Fermentas (Vilnius, Lithuania). SYBR Premix Ex $\operatorname{Taq}^{\mathrm{Tm}}$ II was from TaKaRa (Dalian, China).

Table 1 Sequences of real-time PCR primers

\begin{tabular}{lll}
\hline Genes & Primers & \\
\hline GAPDH & $F\left(5^{\prime}-3^{\prime}\right)$ & TCA ACG GCA CAG TCA AGG \\
IL-6 & $R\left(5^{\prime}-3^{\prime}\right)$ & ACT CCA CGA CAT ACT CAG C \\
& $F\left(5^{\prime}-3^{\prime}\right)$ & AGC CAG AGT CCT TCA GAG AGA TAC \\
TNF-a & $R\left(5^{\prime}-3^{\prime}\right)$ & AAT TGG ATG GTC TTG GTC CTT AGC \\
& $F\left(5^{\prime}-3^{\prime}\right)$ & GCT CT CTG TCT ACT GAA CTT CGG \\
iNOS & $R\left(5^{\prime}-3^{\prime}\right)$ & ATG ATC TGA GTG TGA GGG TCT GG \\
& $F\left(5^{\prime}-3^{\prime}\right)$ & CCC TTC CGA AGT TTC TGG CAG CAG C \\
IL-10 & $R\left(5^{\prime}-3^{\prime}\right)$ & CCA AAG CCA CGA GGC TCT GAC AGC C \\
& $F\left(5^{\prime}-3^{\prime}\right)$ & GGT TGC CAA GCC TTA TCG TA \\
Arg-1 & $R\left(5^{\prime}-3^{\prime}\right)$ & ACC TGC TCC ACT GCC TTG CT \\
& $F\left(5^{\prime}-3^{\prime}\right)$ & ATG CTC ACA CTG ACA TCA ACA CTC \\
MMR & $R\left(5^{\prime}-3^{\prime}\right)$ & CTC TTC CAT CAC CTT GCC AAT CC \\
& $F\left(5^{\prime}-3^{\prime}\right)$ & CAT GAG GCT TCT CCT GCT TCT G \\
& $R\left(5^{\prime}-3^{\prime}\right)$ & TTG CCG TCT GAA CTG AGA TGG \\
\hline
\end{tabular}

F: forward primer; R: reverse primer.

\section{RNA isolation and real-time PCR}

Total RNA was isolated with the RNA Fast 200 kit according to the manufacturer's instructions. First-strand cDNA was synthesized with the RevertAid ${ }^{\mathrm{Tm}}$ First Strand cDNA Synthesis Kit and analyzed by real-time quantitative PCR with SYBR Premix Ex Taq ${ }^{\text {TM }}$ II. PCR was performed on the $\mathrm{IQ}^{\mathrm{TM}}$ Multicolor real-time PCR and detection system (Bio-Rad, Hercules, CA, USA). Data were analyzed on the basis of relative expression method using the formula $\Delta \Delta C$ expression $=2^{-\Delta \Delta \mathrm{Ct}}$, where $\Delta \Delta \mathrm{Ct}=\Delta \mathrm{Ct}$ (treated group) $\Delta \mathrm{Ct}$ (control group), $\Delta \mathrm{Ct}=\mathrm{Ct}$ (target gene) $-\mathrm{Ct}(\mathrm{GAPDH})$, and $\mathrm{Ct}=$ cycle at which the threshold is reached.

\section{Statistical analysis}

Data were analyzed with SPSS for Windows (version 18.0) using one-way ANOVA. Unless otherwise stated, graphs show mean \pm SD. For each dataset, $p<0.05$ was considered statistically significant.

\section{Results}

Effects of Hcy and LPS on mRNA expression in macrophages Different concentrations of Hcy (0, 20, 50 and $100 \mu \mathrm{mol} / \mathrm{L})$ combined with a fixed concentration of LPS $(200 \mathrm{ng} / \mathrm{mL})$ were tested. The mRNA levels of M1 markers (IL-6, TNF- $\alpha$, and iNOS) were up-regulated by the 12-hour treatment with different concentrations of Hcy combined with LPS. mRNA expression levels of these genes reached their maxima at an Hcy concentration of $50 \mu \mathrm{mol} / \mathrm{L}$ (Figure 1). Expression of M2 marker genes did not change significantly in response to the combined Hcy/LPS treatment (data not shown).

\section{Time effect of Hcy and LPS treatment on mRNA expression in macrophages}

M1-associated genes reached their highest mRNA expression levels at an Hcy concentration of $50 \mu \mathrm{mol} / \mathrm{L}$. To determine if Hcy affects expression of M1-associated genes in a time-dependent manner, we measured the corresponding mRNA transcript levels at different time points. When cells were treated with $50 \mu \mathrm{mol} / \mathrm{L}$ Hcy and $200 \mathrm{ng} / \mathrm{mL}$ LPS, mRNA expression of IL-6, TNF- $\alpha$, and iNOS peaked after 12, 24, and 24 hours, respectively (Figure 2).

\section{Effects of Hcy and LPS on mRNA expression in M2 macrophages}

Because the combination of Hcy and LPS could induce macrophage differentiation towards the M1 subtype, we were compelled to investigate if similar treatment could convert the M2 subtype into M1. RAW264.7 macrophages were first induced into the M2 subtype using IL-4 (10 ng/mL) for 12 hours. The three M2 markers (IL-10, Arg-1, and MMR) were up-regulated, whereas the M1 markers (IL-6, TNF- $\alpha$, and iNOS) were downregulated, indicating the successful polarization to M2. 

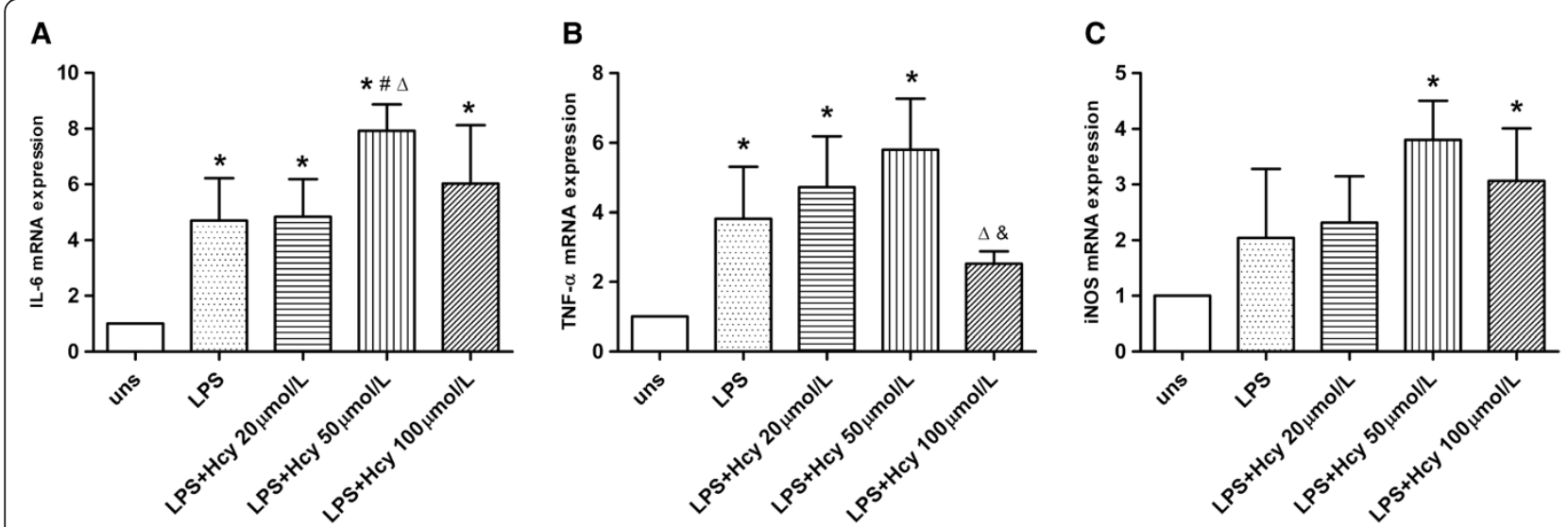

Figure 1 Effects of Hcy and LPS on mRNA expression of M1 marker genes. (A) IL-6, (B) TNF-a and (C) iNOS mRNA expression at $12 \mathrm{~h}$ were assessed by real-time PCR. Gene expression is represented as fold-change compared to unstimulated macrophage. Data are representative of three independent experiments. (uns: unstimulated macrophages; ${ }^{*} p<0.05$ vs uns, $\# p<0.05$ vs LPS, $\Delta p<0.05$ vs LPS + Hcy $20 \mu \mathrm{mol} / \mathrm{L}, \& p<0.05$ vs LPS + Hcy 50 umol/L).

Subsequently, these M2 cell were co-cultured with different concentrations of Hcy and a fixed concentration for 12 hours. After treatment, the gene expression of M2 markers decreased, whereas the gene expression of M1 markers increased (Figure 3).

\section{Time effect of Hcy and LPS on mRNA expression in M2 macrophages}

To find out whether the effect of combined Hcy and LPS treatment on mRNA expression in M2 macrophages is time-dependent, a combination of $50 \mu \mathrm{mol} / \mathrm{L}$ Hcy and LPS $(200 \mathrm{ng} / \mathrm{mL})$ was used to treat M2 macrophages arising from IL-4 induction. mRNA expression levels of IL-6, TNF- $\alpha$, and iNOS reached their maxima after 6 , 12 , and 12 hours, respectively. In contrast, the expression of IL-10, Arg-1, and MMR declined after prolonged incubation with the combined treatment (Figure 4).

\section{Discussion}

Macrophages are considered to be important immune effector cells. Recent studies have uncovered plasticity and heterogeneity in their differentiation pathways, as demonstrated by changes in cellular phenotype and physiology in response to micro-environmental stimuli. Mirroring the Th1/Th2 nomenclature, polarized macrophages are categorized into M1 and M2 subtypes. The classical subtype of activated macrophages, M1, can initiate adaptive immune responses and produce various pro-inflammatory cytokines. These biochemical responses are often involved in killing microorganisms and tumor cells. Moreover, M1 macrophages can promote atherosclerosis (AS). The alternative activated subtype, M2, also plays various roles, such as directing Th2 humoral response, killing parasites, repairing tissues, and producing anti-inflammatory cytokines. M2 macrophages are regarded as inhibitors of AS. However, it is noteworthy that the distinction between M1 and M2 macrophages may not be absolutely definitive; many researchers consider the M1 and M2 subtypes as opposing extremes of the macrophage spectrum [13].

The classical activation of M1 macrophages by interferon$\gamma$ (IFN- $\gamma$ ) and LPS creates cells that can produce various pro-inflammatory chemokines and cytokines such as IL-6

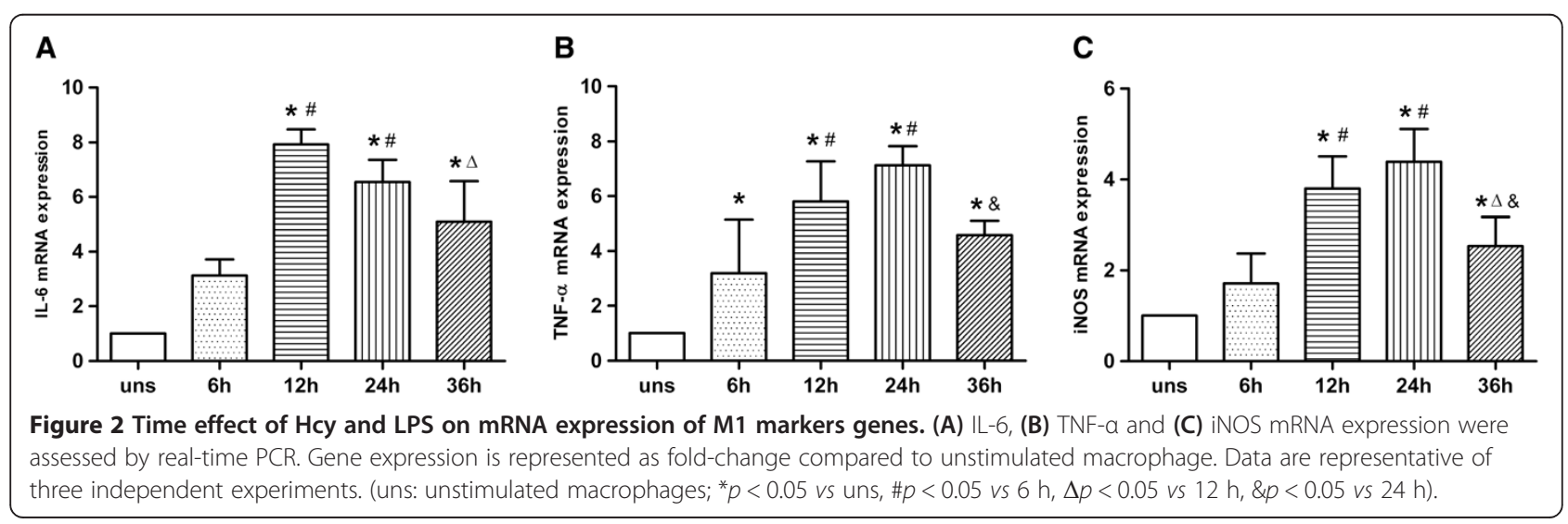




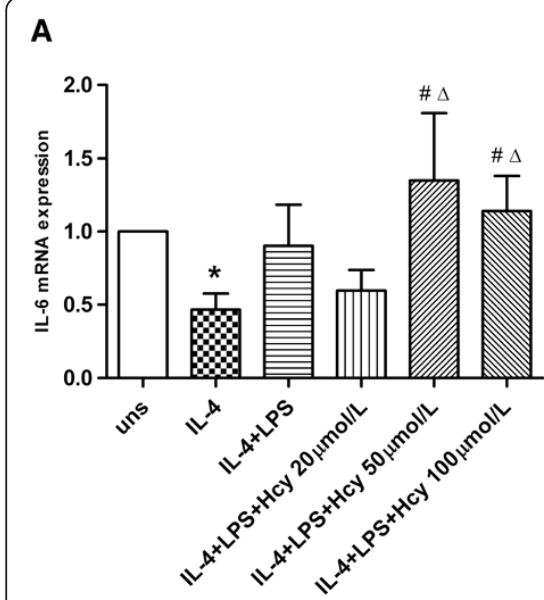

D

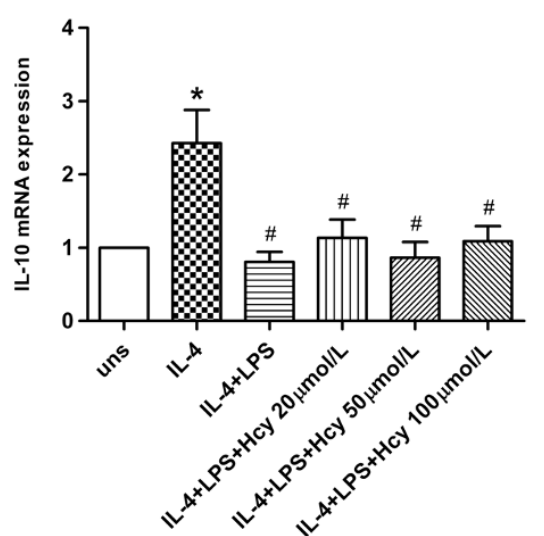

B

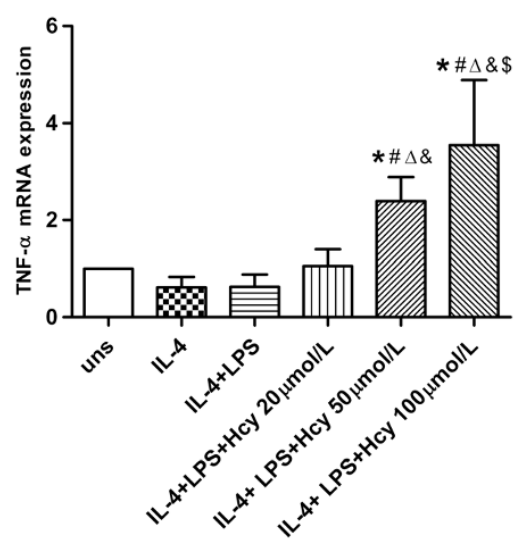

E

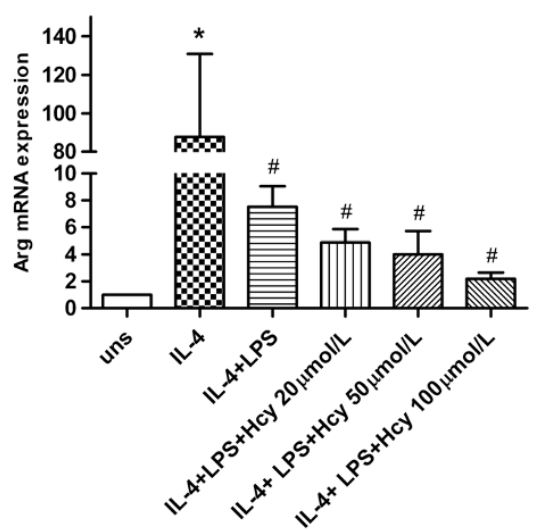

C

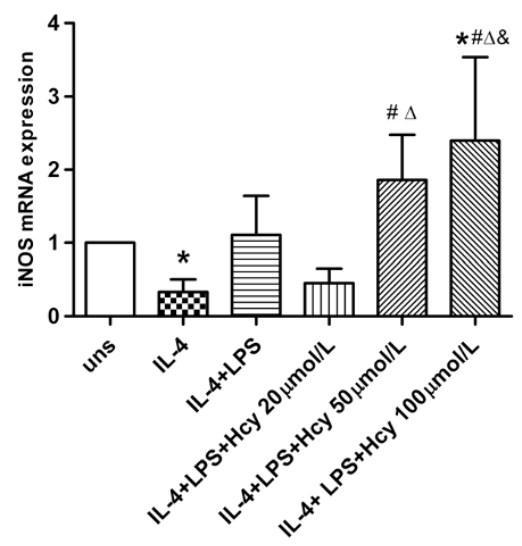

$\mathbf{F}$

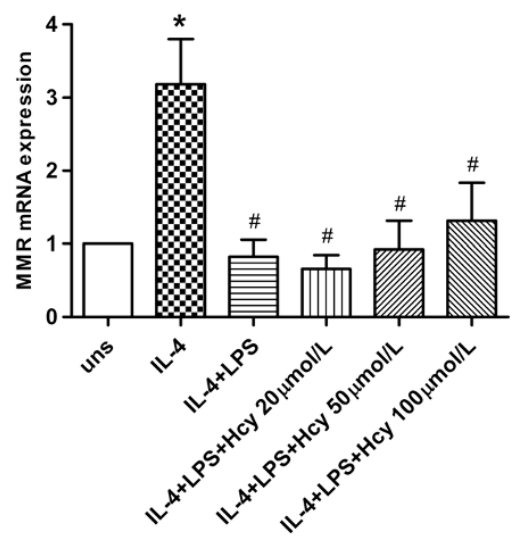

Figure 3 Effects of Hcy and LPS on mRNA expression in M2 macrophage. (A) IL-6, (B) TNF-a, (C) iNOS, (D) IL-10, (E) Arg-1 and (F) MMR mRNA expression were assessed by real-time PCR. Gene expression is represented as fold-change compared to unstimulated macrophages. Data are representative of three independent experiments. (uns: unstimulated macrophages; Arg stands for Arg-1; ${ }^{*} p<0.05$ vs uns; \#p $<0.05$ vs IL-4; $\Delta p<0.05$ vs LPS; \&p $<0.05$ vs LPS + Hcy $20 \mu \mathrm{mol} / \mathrm{L} ; \$ p<0.05$ vs LPS + Hcy $50 \mu \mathrm{mol} / \mathrm{L})$.

and TNF- $\alpha$. M2 macrophages, usually activated by IL-4, produce anti-inflammatory cytokines such as IL-10. Aside from their differential expression of opposing effector molecules, inducible nitric oxide synthase (iNOS) is expressed more in M1 macrophages, whereas arginase-1 (Arg-1) and mannose membrane receptor (MMR) are expressed more in M2 macrophages, reflecting the different immunological roles of these two populations [7]. In this study, we chose IL-6, TNF- $\alpha$, and iNOS as M1 macrophage markers, whose expression was induced by IFN- $\gamma$ and LPS. We chose IL-10, Arg-1, and MMR as M2 macrophage markers, whose expression was induced by IL-4. These results are consistent with our previous work [12].

LPS, the primary glycolipid component found in the outer membranes of Gram-negative bacteria, can induce inflammatory responses, activate the complement system, and initiate the coagulation pathway [14]. Many studies have confirmed that in the presence of IL-1 or IFN- $\gamma$, the
LPS-induced defense reaction and killing ability could be augmented. Conversely, the pro-inflammatory effect of IFN- $\gamma$ can be enhanced by the presence of LPS $[15,16]$. Because we had been unable to stimulate macrophage subtype polarization using Hcy alone (data not shown), we decided to use a combination of Hcy and LPS.

In the presence of LPS, Hcy led to up-regulated expression of M1 macrophage markers (IL-6, TNF- $\alpha$, and iNOS); the optimal Hcy concentration was $50 \mu \mathrm{mol} / \mathrm{L}$. However, Hcy and LPS elicited no significant effects on the expression of M2 macrophage markers (IL-10, Arg-1, and MMR). No correlation between the concentration of Hcy and the expression of marker genes was observed. This was possibly due to higher Hcy concentrations leading to more cell death. We also found that a combination of Hcy and LPS could transform M2 subtype into M1. This phenomenon may help to explain the pro-atherosclerotic effects of Hcy. 


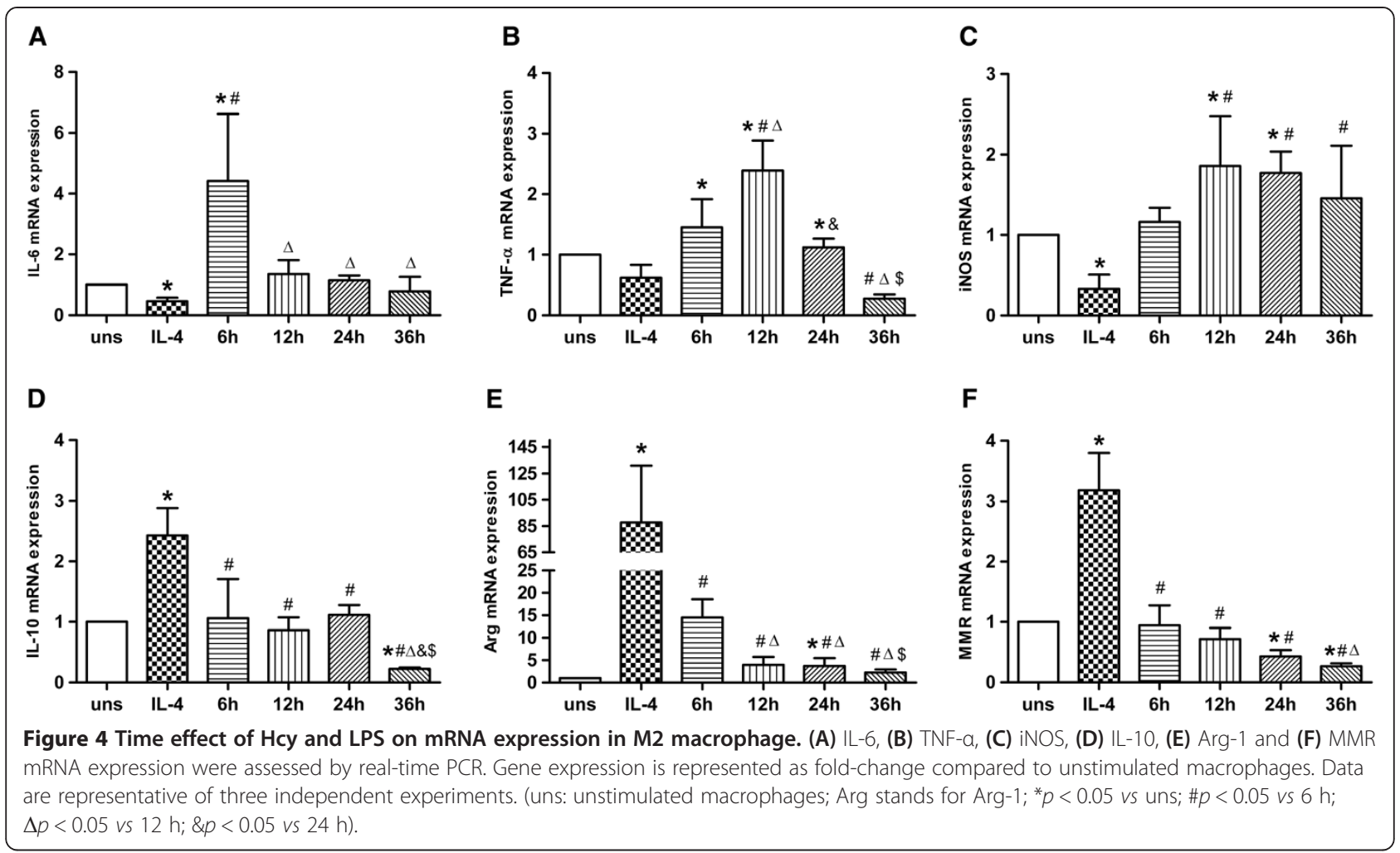

Many studies have shown that Hcy can act as a promoter of AS by activating inflammatory immune reactions. The immuno-inflammation hypothesis is a generally accepted explanation for the mechanism of AS, and macrophages are believed to be important in this process. The differentiation of macrophages into different sub-populations has become a new exploratory area in the initiation and progression of AS. Zhang et al. have demonstrated that Hyperhomocysteinemia (HHcy) could accelerate AS and enhance Ly-6C Monocyte/Macrophage accumulation in the lesions of $\mathrm{Tg}$-hCBS apoE ${ }^{-1-} \mathrm{Cbs}^{-1-}$ mice. They also found that monocyte/macrophage accumulation in the lesions of Ly-6C-positive mice correlated positively with Hcy levels. However, little is known about the influence of Hcy on the polarization of cultured macrophages [17]. In this study, we used a cell-based assay to demonstrate that Hcy could induce macrophage polarization into the pro-inflammatory phenotype, thereby providing further evidence in support of the model for Hcy-induced AS.

\section{Conclusions}

We investigated the synergistic effects of Hcy and LPS on the differentiation and conversion of RAW264.7 macrophages. The results showed that a combination of Hcy and LPS could induce macrophages to differentiate into the pro-inflammatory subtype. The differentiated anti-inflammatory subtype could also be converted to the pro-inflammatory subtype by combined treatment with Hcy and LPS. These findings provide new evidence for the role of Hcy in facilitating the progression of AS.

\section{Abbreviations}

AS: Atherosclerosis; Hcy: Homocysteine; HHcy: Hyperhomocysteinemia; IL-6: Interleukin-6; IL-10: Interleukin-10; LPS: Lipopolysaccharide; iNOS: Inducible nitric oxide synthase; IFN- $\gamma$ : Interferon- $\gamma$; Arg-1: Arginase-1; MMR: Mannose membrane receptor.

\section{Competing interests}

The authors declare that they have no competing interests.

\section{Authors' contributions}

SG carried out the main body of this work and drafted the manuscript. WL, YW and LW participated in designing the studies. ZY participated in the design and coordination and helped to draft the manuscript. All authors have read and approved the final manuscript.

\section{Sources of funding}

This work was supported in part by the National Science Fund for Distinguished Young Scholars (81025002 to ZY) and the Natural Science Foundation of China (30871043 to ZY and 81100209 to YW).

Received: 4 March 2013 Accepted: 8 May 2014

Published: 13 May 2014

\section{References}

1. Galkina E, Ley K: Immune and inflammatory mechanisms of atherosclerosis. Annu Rev Immunol 2009, 27:165.

2. Ross R: Atherosclerosis is an inflammatory disease. Am Heart J 1999, 138:419-420.

3. Libby P, Ridker PM, Maseri A: Inflammation and atherosclerosis. Circulation 2002, 105(9):1135-1143.

4. Linton MRF, Fazio S: Macrophages, inflammation, and atherosclerosis. Int J Obes 2003, 27:S35-540. 
5. Weber C, Zernecke A, Libby P: The multifaceted contributions of leukocyte subsets to atherosclerosis: lessons from mouse models. Nat Rev Immunol 2008, 8(10):802-815

6. Gordon S, Martinez FO: Alternative activation of macrophages: mechanism and functions. Immunity 2010, 32(5):593-604.

7. Mantovani A, Sica A, Sozzani S, Allavena P, Vecchi A, Locati M: The chemokine system in diverse forms of macrophage activation and polarization. Trends Immunol 2004, 25(12):677-686.

8. Hofmann MA, Lalla E, Lu Y, Gleason MR, Wolf BM, Tanji N, Ferran $L J$ Jr, Kohl B, Rao V, Kisiel W, Stern DM, Schmidt AM: Hyperhomocysteinemia enhances vascular inflammation and accelerates atherosclerosis in a murine model. J Clin Invest 2001, 107(6):675-683.

9. de Lawrence Koning A, Werstuck GH, Zhou J, Austin RC: Hyperhomocysteinemia and its role in the development of atherosclerosis. Clin Biochem 2003, 36(6):431-441.

10. Austin RC, Lentz SR, Werstuck GH: Role of hyperhomocysteinemia in endothelial dysfunction and atherothrombotic disease. Cell Death Differ 2004, 11(Suppl 1):S56-S64.

11. Zhang D, Fang P, Jiang X, Nelson J, Moore JK, Kruger WD, Berretta RM, Houser SR, Yang X, Wang H: Severe hyperhomocysteinemia promotes bone marrow-derived and resident inflammatory monocyte differentiation and atherosclerosis in LDLr/CBS-deficient mice. Circ Res 2012, 111(1):37-49.

12. Chen T, Liang $X$, He M, Wu Y, Yuan Z: Introduction and Identification of M1/M2 Phenotypes of RAW264.7. Mol Cardiol China 2011, 2:39-42.

13. Edwards JP: Exploring the full spectrum of macrophage activation Nat Rev Immunol 2008, 8(12):958-969.

14. Fujihara M, Muroi M, Tanamoto K, Suzuki T, Azuma H, Ikeda H: Molecular mechanisms of macrophage activation and deactivation by lipopolysaccharide: roles of the receptor complex. Pharmacol Ther 2003, 100(2):171-194

15. Wan Y, Freeswick PD, Khemlani LS, Kispert PH, Wang SC, Su GL, Billiar TR: Role of lipopolysaccharide (LPS), interleukin-1, interleukin-6, tumor necrosis factor, and dexamethasone in regulation of LPS-binding protein expression in normal hepatocytes and hepatocytes from LPS-treated rats. Infect Immun 1995, 63(7):2435-2442.

16. Adams DO, Hamilton TA: The cell biology of macrophage activation. Annu Rev Immunol 1984, 2(1):283-318.

17. Zhang D, Jiang X, Fang P, Yan Y, Song J, Gupta S, Schafer Al, Durante W, Kruger WD, Yang $X$, Wang H: Hyperhomocysteinemia promotes inflammatory monocyte generation and accelerates athrosclerosis in transgenic cystathionine beta-synthase-deficient mice. Circulation 2009, 120(19):1893-1902.

doi:10.1186/1476-9255-11-13

Cite this article as: Gao et al:: The synergistic effect of homocysteine and lipopolysaccharide on the differentiation and conversion of raw264.7 macrophages. Journal of Inflammation 2014 11:13.

\section{Submit your next manuscript to BioMed Central and take full advantage of:}

- Convenient online submission

- Thorough peer review

- No space constraints or color figure charges

- Immediate publication on acceptance

- Inclusion in PubMed, CAS, Scopus and Google Scholar

- Research which is freely available for redistribution

Submit your manuscript at www.biomedcentral.com/submit
C BioMed Central 